\title{
Comparative Transcriptomic Analysis of Two Cucumis Melo Var. Saccharinus Germplasms Differing in Fruit Physical and Chemical Characteristics
}

\section{Renfan Liang ( $\sim$ gxnkylrf@gxaas.net)}

Guangxi Academy of Agricultural Sciences

\section{Yicheng Su}

Guangxi Academy of Agricultural Sciences

\section{Xiaojuan Qin}

Guangxi Academy of Agricultural Sciences

\section{Zhongkui Gao}

Guangxi Academy of Agricultural Sciences

\section{Zhixin Fu}

Guangxi Academy of Agricultural Sciences

Huijun Qiu

Guangxi Academy of Agricultural Sciences

\section{Xu Lin}

Guangxi Academy of Agricultural Sciences

\section{Jinlian Zhu}

Guangxi Normal University for Nationalities

\section{Research Article}

Keywords: Cucumis melo var. saccharinus, soluble sugar, physical characteristics, fruit ripening, transcriptome

Posted Date: December 15th, 2021

DOI: https://doi.org/10.21203/rs.3.rs-1109979/v1

License: (1) This work is licensed under a Creative Commons Attribution 4.0 International License. Read Full License 
Version of Record: A version of this preprint was published at BMC Plant Biology on April 12th, 2022. See the published version at https://doi.org/10.1186/s12870-022-03550-8. 


\section{Abstract}

Background: Hami melon (Cucumis melo var. saccharinus) is a popular fruit in China noted for its excellent taste, which is largely determined by its physical and chemical characteristics, including flesh texture, sugar content, aroma, and nutrient composition. However, the mechanisms through which the associated with these characteristics are regulated have not yet been sufficiently determined. In this study, we monitored changes in the fruits of two germplasms differing in physical and chemical characteristics throughout the period of fruit development.

Results: Ripe fruit of the bred variety 'Guimi' had significantly higher soluble sugar contents than the fruit of the common variety 'Yaolong', whereas differences in fruit shape and color between these two germplasms were observed during the course of development. Comparative transcriptome analysis, conducted to identify regulators and pathways underlying the observed differences at corresponding stages of development, revealed a higher number of differentially expressed genes (DEGs) in Guimi than in Yaolong. Moreover, most of the DEGs detected during early fruit development of Guimi were associated with cell wall biogenesis. Temporal analysis of the identified DEGs revealed similar trends in the enrichment of downregulated genes in both germplasms, although there were certain differences in the enrichment trends of upregulated genes. Further analyses revealed trends in the differential changes of multiple genes involved in cell wall biogenesis and sugar metabolism during fruit ripening.

Conclusions: We were thus able to identify a number of genes associated with the ripening of Hami melon, which will accordingly provide novel insights into the molecular mechanisms underlying the development of fruit characteristics in these melons.

\section{Background}

Hami melon (Cucumis melo var. saccharinus) is among the most important diploid crops within the family Cucurbitaceae, the different cultivars of which are characterized by highly variable fruit traits, such as flesh color, sugar contents, and shape (Wang et al. 2018) [1]. Production of Hami melons in Guangxi Province, China, started in the middle to late 1990s (Nunez-Palenius et al. 2008). However, given the small differences in day and night temperatures, the Guangxi environment is not particularly conducive to sugar accumulation in Hami melon, as it is difficult to achieve the desirable 14\% soluble solid content (Bi et al. 2003). In addition, high temperatures and humidity often contribute to the development of a range of diseases and pest infestations, thereby reducing yields and resulting in heavy economic losses, notably as a consequence of gummy stem blight caused by Mycosphaerella melonis. To address these problems, 'Guimi-12', a new high-quality Guangxi germplasm, characterized by high yields and disease resistance, has been selected and popularized. Guimi-12 differs from the common Hami melon germplasm ('Yaolong') with respect to shape, size, flesh texture, sugar content, aroma, and nutrient composition, which are acknowledged to be important considerations in breeding of fruit-bearing plants and are typically the focus of studies seeking to identify and characterize the associated regulatory transcription factors and molecular mechanisms (Guo et al. 2015). At present, however, little is known about the 
differences between these two Hami melon varieties with respect to gene expression patterns and regulatory pathways.

To date, breeding programs for Hami melon have tended to focus primarily on the selection of traits associated with fruit thickness and sweetness (Yu et al. 2012). Pericarp hardening is generally associated with the development of secondary cell wall structures, although the underlying mechanisms have yet to be ascertained. In this regard, however, it has been established that a transcription network comprising NAC and MYB transcription factors is highly conserved in the regulation of pericarp formation and secondary cell wall development in Arabidopsis (Dardick et al. 2010). With respect to sweetness, it has been established that sucrose is the predominant sugar in ripe melon fruit (Burger et al., 2003; Villanueva et al., 2004). a-Galactosidase (a-Gal) has been identified as the initial enzyme in the decomposition of stachyose and its activity has been shown to be closely associated with the accumulation of fructose and glucose during fruit expansion (Alan et al. 2007). Consistently, acidic a-Gal has been found to have higher activity in the sweet tissue of watermelon fruit than that in unsweet tissue, thereby highlighting its significance in the accumulation of sugar in watermelon (Colla et al. 2006). Numerous metabolic pathways have been shown to contribute to sugar accumulation in watermelon fruit (Braun et al. 2012), with 62 sugar synthesis enzymes and 76 sugar transporter genes associated with these pathways having been identified (Guo et al. 2013), 13 and 14 genes of which, respectively, have been shown to be differentially expressed during fruit development (Liu et al. 2013). In addition, it has also been found that the patterns of sugar accumulation during fruit maturation differ between oriental and other types of melon (Saladie et al. 2015). These observations thus indicate that differences in the genes and pathways associated sugar metabolism probably determine the differing sucrose contents of the fruits, and we accordingly speculate that sugar contents in the fruit of Guimi-12 Hami melon might also differ from those in Yaolong Hami melon.

Transcriptomes are collections of all transcripts produced by a species or specific types of organs, tissues, and cells, and include the number of transcripts, expressional dynamics at specific developmental stages, post transcriptional modification, and regulation of non-coding RNAs (Ashburner et al. 2000). By conducting transcriptomic studies, we can perform more comprehensive analyses of gene expression, structure, and function in specific species, and thereby gain a more complete understanding of the molecular mechanism underlying specific physiological characteristics (Pruitt et al. 2005). Compared with the whole genome, properties at the transcriptome level tend to be more temporally and spatially discrete, as has been reported in numerous studies (Trapnell et al. 2010; Grassi et al. 2013). Thus, transcriptome sequencing technology can provide a new approach to the study of gene function and physiological characteristics in Hami melon. In this study, we sought to comparatively analyze the transcriptomes of two contrasting Hami melon germplasms, namely, Guimi-12 and Yaolong, throughout the fruit ripening process, with the aim of identifying those genes playing roles in the development and ripening of Hami melon fruit, and thereby provide further insights for identifying key pathways and regulators associated with the physical and chemical characteristics of Hami melon fruit. 


\section{Methods}

\section{Plant materials}

The germplasm 'Guimi' was provided by Vegetable Research Institute, Guangxi Academy of Agricultural Sciences, Nanning, China. The germplasm 'Yaolong' was purchased from Fuyou Seedlings Co. LTD, Hainan, China. Both germplasms were cultivated in a greenhouse, in which the temperature was maintained between $25^{\circ} \mathrm{C}$ to $30^{\circ} \mathrm{C}$ during the day and above $15^{\circ} \mathrm{C}$ at night. Flowers were manually pollinated, and three to five biological replicate fruits were harvested from selected plants at $10,20,30$, and 40 DAP. These fruits were frozen in liquid nitrogen and stored at $-80^{\circ} \mathrm{C}$ until used for analyses.

\section{Determination of physical and chemical characteristics}

Among the physical characteristics measured, the size and weight of fruit were measured at $5,10,15,20$, $25,30,35$, and 40 DAP. The Brix value of fruit was determined using an ATAGO PAL-2 refractometer, as previously described (Abbas et al. 2020). Each measurement was conducted for least three replicates. The soluble sugar contents (total soluble solids, glucose, fructose, and sucrose) were analyzed spectrophotometrically, as described previously (Zhu et al. 2017).

\section{RNA isolation, cDNA library preparation, and RNA-Seq}

Total RNA was extracted from the pulp of frozen fruit collected at each time point using a TRIzol reagent kit (Invitrogen, Carlsbad, CA, USA) based on the manufacturer's protocol, and the quality of the isolated total RNA was evaluated using an Agilent 2100 Bioanalyzer (Agilent Technologies, Palo Alto, CA, USA) and RNase-free agarose gel electrophoresis. mRNA was enriched, fragmented, and reverse transcribed to first-strand cDNA using Oligo(dT), fragmentation buffer, and random primers. Second-strand cDNA was subsequently synthesized using DNA polymerase I, RNase H, dNTPs, and buffer. Finally, 24 cDNA libraries were constructed and sequenced using the Illumina Novaseq6000 platform with pair-end 150bp mode.

\section{Global and differential gene expression analysis}

To obtain high-quality clean reads, reads containing adapter and poly-N sequences or low-quality bases were filtered out using fastp (version 0.18.0) (Chen et al., 2018) with default parameters. Thereafter, the rRNA mapped reads were removed following the alignment of clean reads to a ribosomal RNA (rRNA) database using Bowtie2(version2.28) (Langmead and Salzberg, 2012). As a Cucumis melo reference genome, we used the RefSeq assembly accession GCF_000313046.1. The remaining cleaned reads were aligned to the reference genome with HISAT2 (version 2.4) using "-rna-strandness RF" (Kim et al., 2015), and genes were assembled using StringTie (version 1.3.1) based on these mapped reads (Pertea et al., 2015). RSEM software were used to calculate gene expression values based on well-mapped reads and normalized to the FPKM (the fragments per kilobase of exon per million mapped fragments) values, as previously described (Ma et al., 2020). 
For analysis of differential gene expression between the Yaolong and Guimi groups, we used DESeq2 software. Differentially expressed genes (DEGs) were identified based on the criteria of a false discovery rate $(\mathrm{FDR})<0.05$ and an absolute value of log2(fold change) $\geq 1$.

\section{Temporal analysis}

To determine trends in the changes in gene expression during $C$. melo fruit development, we performed temporal analysis for the DEGs of each variety using Short Time-series Expression Miner (STEM) software with default parameters (Ernst et al. 2006). Genes were profiled by clustering based on the corresponding $P$-values and those with a $P$-value $\leq 0.05$ were considered differentially expressed, These DEGs were subjected to GO term and KEGG pathway enrichment analyses using hypergeometric distribution tests. GO terms and KEGG pathways with Q-values equal to or less than 0.05 were considered functional annotations.

\section{Results}

\section{Variations in the physical and chemical characteristics of melon fruit during ripening}

For comparisons of fruits of the two assessed Hami melon varieties, we examined the physiochemical characteristics of fruit collected at different stages of development (Fig. 1). The color of the fruit peel of the two varieties was found to be similar at 5 and 10 days after pollination (DAP). Thereafter, however, the peel of Yaolong fruit turned a darker green compared with that of Guimi from 15 to 20 DAP. With continued growth, Guimi melons developed as medium-sized oval fruits with a yellow skin. In both varieties, the juicy flesh underwent a change in color from white to yellow during ripening.

Trends in the changes in physical and chemical characteristics of the fruits, including weight, size, and soluble sugar contents, are shown in Fig. 2. Similar trends in the two varieties were observed with respect to the weight and size of fruit during repining. Interestingly, fruit weight increased rapidly from 35 to 40 DAP, whereas there were only slight increases in size. At 40 DAP, the soluble sugar contents of Guimi fruit had reached a value 12, which was higher than that obtained for Yaolong, although differences in the brix values of the two varieties were found to be non-significant.

\section{Global analysis of the RNA-Seq data}

To determine gene expression patterns, we performed RNA-seq analysis using the $C$. melo reference genome. After filtering out rRNAs and low-quality reads, a total of 111 million reads were mapped to the reference genome (Table S1). For these clean reads, we obtained average mapped reads per sample greater that $90 \%$. The one exception in this the regard was the low $(74.44 \%)$ alignment ratio obtained for Yaolong at 40 DAP. In total, we detected 21,172 expressed genes among the melon fruit samples.

To determine differences in the expression of genes between two close sampling times, we identified the DEGs in Guimi and Yaolong based on the threshold criteria of a log2 fold change $\geq 1$ and FDR $\leq 0.05$ (Table S2). For this purpose, we defined the earliest time point of paired groups as the control sample for 
subsequent measurements. The number of DEGs in Guimi was found to be markedly higher than that in Yaolong at 20 and 30 DAP, while DEGs being identified in Guimi less than Yaolong (223 vs 95) at 40 DAP (Fig. 3A). These finding would thus appear to indicate that during fruit development the constituents of Guimi fruit undergo more pronounced changes than those of Yaolong fruit. Over time, however, there was a gradual reduction in the number of DEGs in both melon varieties during the fruit development, indicting a corresponding reduction in the speed of fruit growth and that the fruit was fully mature at 40 DAP. For both Guimi and Yaolong, we also detected perturbations in gene expression at the same time points. The number of genes differentially expressed between Guimi and Yaolong initially increased, reaching a peak at 20 DAP, after which the number declined to 458 at 40 DAP (Fig. 3B).

For the purpose of functional annotation, identified DEGs in the two melon varieties were assigned to GO terms and KEGG pathways. We accordingly found that for Yaolong melon, the majority of enriched GO terms between 10 and 20 DAP were classified in the category biological process, including 'histone lysine methylation', 'peptidyl-lysine methylation', and 'DNA alkylation', whereas in the case of Guimi melon, the most significantly enriched GO terms were 'polysaccharide metabolism', 'cell wall biogenesis', and 'external encapsulating structure organization' (Fig. S1 and S2). For the second comparison (20 DAP vs. 30 DAP), the DEGs in Guimi melon were found to be significant enriched in the processes of 'response to acid chemical', 'plant-type cell wall biogenesis', and 'response to chemical', whereas for Yaolong, the three most enriched biological processes were 'hemicellulose metabolism', xylan metabolism', and 'cell wall polysaccharide metabolism'. Comparing between 30 and 40 DAP samples revealed that the DEGs of Guimi melon were notably enriched with respect to 'amine metabolism', 'cellular amine metabolism', and 'jasmonic acid metabolism', whereas those of Yaolong melon were found to be enriched in the cellular component categories 'external encapsulating structure', 'cell periphery', and 'vacuole'. With regards to KEGG pathway annotation, we found that in the 10 to 20 DAP comparison, DEGs in Yaolong were enriched with respect to 'DNA replication', 'ABC transporters', and 'flavone and flavonol biosynthesis', whereas 'metabolic pathways', 'biosynthesis of secondary metabolites', and 'phenylpropanoid biosynthesis' were found to be enriched with Guimi DEGs (Fig. S3 and S4). Interestingly, for the 20 to 30 DAP comparison, we observed that for both melon varieties, the three most significantly DEG-enriched KEGG pathways were 'biosynthesis of secondary metabolites', 'metabolic pathways' and 'phenylpropanoid biosynthesis'. For the third comparison between 30 and 40 DAP, Guimi DEGs were found to be enriched in pathways such as 'valine, leucine, and isoleucine biosynthesis', 'biosynthesis of secondary metabolites' and 'cyanoamino acid metabolism', whereas pathways enriched with Yaolong DEGs included 'biosynthesis of secondary metabolites' and 'metabolic pathways'.

\section{Comparison of trends in temporal gene expression during melon fruit ripening}

To gain further insights into the change trends in gene expression during fruit development, we clustered a total of 4,731 DEGs from Guimi melon and 3,198 DEGs from Yaolong into 38 profiles using the STEM algorithm. Among these, 2,897 Guimi DEGs were significantly clustered into the following six profiles: two upregulated profiles (Profiles 17 and 12), three downregulated profiles (Profiles 0, 7, and 2), and one biphasic expression pattern profile (Profile 18) (Fig. 4A). Similarly, 2,217 Yaolong DEGs were classified 
into the following six profiles based on $P$ values $\leq 0.05$ : two upregulated patterns, one biphasic expression pattern, and three downregulated patterns (Fig. 4B).

To systematically investigate the biological functions of candidate genes, we extracted DEGs from upand downregulated cluster groups for further GO term and KEGG pathway analyses. GO analysis revealed that Yaolong DEGs assigned to profiles 17 and 19 were separately categorized into 18 biological processes and two molecular processes, whereas Guimi DEGs in profile 12 were enriched with respect to 110 major functions in the Biological process, Cellular component, and Molecular function categories (Fig. S5). However, we detected no significant enrichment of the Guimi DEGs clustered in profile 17. The GO terms with most representation for downregulated cluster groups are shown in Fig. S6. Among biological functions, 'gibberellin metabolic process' (G0:0009685), 'cytoskeleton' (GO:0005856), and 'cell wall organization or biogenesis' (G0:0071554) were the most significantly enriched functions in Guimi profiles 7, 2, and 0, respectively. For profile 0, 2, and 7 of Yaolong DEGs, 'cell wall' (G0:0005618), 'histone lysine methylation' (GO:0034968), and 'phenylpropanoid biosynthetic process' (G0:0009699), respectively, were most enriched biological functions.

On the basis of KEGG analysis, we identified nine KEGG pathways enriched with downregulated Guimi DEGs including 'metabolic pathways' (ko01100), 'biosynthesis of secondary metabolites' (ko01110), 'phenylpropanoid biosynthesis' (ko00940), and 'biosynthesis of various secondary metabolites - part 2' (ko00998) (Figure S7). In contrast, only two pathways were enriched with upregulated Guimi DEGs, namely, 'carbon fixation in photosynthetic organisms' (ko00710) and 'galactose metabolism' (ko00052). In total, we identified 12 pathways with significant enrichment of upregulated Guimi DEGs (Fig. S8). These results thus indicate that most of the DEGs regulated during fruit development appear to be associated with the functioning of metabolic pathways.

\section{Analysis of cell wall biogenesis during melon fruit ripening}

Fifty-one DEGs in Guimi, clustered in Profile $0(n=28)$ and Profile $2(n=23)$, were found to be significantly associated with cell wall biogenesis, showing downregulated gene expression patterns, whereas in Yaolong, 48 DEGs showing downregulated trends, clustered into Profiles 0, 2, and 7, showed similar associations. Fig. 5 shows the differences in the expression trends of these DEGs between Guimi and Yaolong. We observed 33 common DEGs in cell wall biogenesis-related profiles of Guimi and Yaolong, a majority of which showed similar patterns of expression regulation in the two melon varieties at different stages of fruit development and ripening (Fig. 5A). For example, gradual reductions in the levels of GUX3 expression were observed during fruit development in both germplasms (Fig. 5B and 5C). A similar reduction in expression was detected for OD01, which peaked during the early stages of fruit development, after which there was a slight reduction, which became more pronounced prior to maturity. Although melon type-specific DEGs involved in cell wall biogenesis showed an overall downregulated pattern, differences in the changes in the direction of gene expression were still different between consecutive stages of development. For example, in Guimi melon, UAM1 was downregulated from stage 1 to 2 , then constantly expressed at a stable level from stage 2 to 3 , prior to undergoing a decline in the 
mature stages. These findings thus indicate differences in the expression patterns of melon cultivarspecific DEGs associated with cell wall synthesis during fruit development.

\section{Analysis of sugar metabolism during melon fruit ripening}

Some of the genes involved in sugar metabolism were found to be differentially expressed during melon fruit development, including those associated with 'galactose metabolism', 'starch and sucrose metabolism', 'fructose and mannose metabolism', and 'amino sugar and nucleotide sugar metabolism' (Fig. 6A). However, in the case of Yaolong, only Profile 0 DEGs were found to be significantly associated with the starch and sucrose metabolism pathway. Contrastingly, in Guimi, 'amino sugar and nucleotide sugar metabolism' was identified as being enriched with Profile 2 DEGs. For a majority of the DEGs associated with the 'amino sugar and nucleotide sugar metabolism' pathway, including MUR4, UGD1, and UPTG2, the lowest levels of expression were detected in ripe fruit (Fig. 6B). Difference in gene expression were also detected during the different stages of fruit development. For example, there was a marked reduction in the expression of GAUT6 during in the middle phase of fruit development, followed by a slight increase in pre-mature fruit, before declining to the lowest levels in mature fruit. Although the change trend in UGD1 expression in young fruit was similar to that observed for GAUT6, it differed in that it was expressed at a constantly stable level in the pre-mature fruit. In Yaolong, a total of 11 Profile 0 DEGs were found to be significantly associated with the starch and sucrose metabolism pathway. In contrast to trends in the expression of sugar metabolism-related genes in Guimi, nine of these DEGs were characterized by gradual downregulated expression trends during fruit development and ripening (Fig. 6C). For example, the lowest level of At4g02290 expression in Yaolong was detected at stage T3. Conversely, however, whereas INV1 was characterized by a downregulated expression trend in young fruit, high levels of expression were detected during the expansion stage, prior to a subsequent decline in expression, reaching the lowest values in ripe fruit.

\section{Discussion}

The trends we observed regarding changes in the physical and chemical characteristics of developing Guimi and Yaolong melon, including weight, size, and soluble sugar contents (Fig. 2), are presume to influence the flavor, taste, storage, and uses of Hami melon (Zhu et al. 2017). Fruit ripening is a complex genetically programmed process, which in Hami melon has not been extensively studied with respect to the underlying molecular mechanisms. To gain a better understanding of the associated gene regulation, we undertook RNA-seq of the Guimi and Yaolong varieties, on the basis of which, we identified 21,172 genes that were expressed among melon fruit samples during different stages development. Of these genes, the numbers of differentially expressed genes in Guimi were found to be markedly higher than those in Yaolong at 20, 30, and 40 DAP (Fig. 3), which would thus tend to indicate that Guimi fruit undergoes more pronounced changes during the course of development, which are assumed to be related to its improved characteristics compared with Yaolong. In maize, analyses at the whole-genome level revealed minimal difference between the drought-tolerant maize inbred line Han21 and drought-sensitive maize inbred line $\mathrm{B} 73$, with respect gene expression levels and the numbers of genes and transcription 
factors. However, when the authors assessed those genes that were differentially expressed between these two lines, they found that in response to drought stress, the number of DEGs in Han21 $(2,832)$ was significantly higher than that in B73 (917) (Zenda et al. 2019; Thirunavukkarasu et al. 2017).

In the present study, we identified polysaccharide metabolism, cell wall biogenesis, and external encapsulating structure organization as the most significantly enriched GO terms among Guimi melon DEGs between 10 and 20 DAP (Fig. S1 and S2). Similarities in differential gene expression patterns between 10 and $20 \mathrm{DAF}$ and 30 and $40 \mathrm{DAF}$ during fruit development have been reported previously, indicating that gene expression is significantly altered during the period from 20 to $30 \mathrm{DAF}$, with implications for fruit development (Zhang et al. 2016). On the basis of our observations, we speculate that differences between Yaolong and Guimi with respect to cell wall biogenesis start to manifest at an early point in fruit development. Moreover, in Guimi melon, we detected differences with respect to a number of metabolic pathways, notably, 'metabolic pathways', 'biosynthesis of secondary metabolites' and 'phenylpropanoid biosynthesis', between 10 and 20 DAP (Figs. S3 and S4), which are probably indicative of the early modification of metabolic regulation during the ripening stage. In this regard, it has been established that plant polysaccharide metabolic pathways play a prominent role in mediating fruit ripening (Yamaguchi et al. 1977).

Melons are consumed for their sweet fruit (Burger et al. 2007), a trait that can be attributed to the accumulation of sucrose during the late stage of fruit development (Schaffer et al. 1987; Hubbard et al. 1989; Schaffer et al. 1996), and in this context, we found that DEGs associated with plant polysaccharide metabolic pathways showed different gene expression patterns during the fruit ripening process, thereby implying a role in the regulation of fruit ripening.

In total, we detected 4,731 and 3,198 genes that were differentially expressed in the Guimi and Yaolong varieties, respectively, during fruit development, which were clustered into 38 profiles based on STEM analysis. Three downregulated profiles (Profiles 0, 2, and 7) were obtained for both Guimi and Yaolong melon, whereas upregulated profiles were notably more prominent in Guimi melon (Fig. 4). In Guimi melon, we identified a number of pathways enriched with upregulated DEGs, namely, 'carbon fixation in photosynthetic organisms' and 'galactose metabolism' (Fig. S8), whereas in contrast, no significantly enriched pathways were identified for the upregulated DEGs of Yaolong melon. The upregulated activity of these pathways has been reported previously and is considered particularly advantageous with respect to the breeding of Hami melons. Carbon fixation pathways are a ubiquitous feature plants, algae, cyanobacteria and other photosynthetic organisms. High $\mathrm{CO}_{2}$ concentrations provide an abundant resource for plant photosynthesis and promote plant growth and biomass accumulation (Xu et al. 2016). Yelle et al. found that in tomato, $\mathrm{CO}_{2}$ can accelerate flower bud differentiation in fruit and vegetable crops, reduce the node position of female flowers, increase the number of female flowers and fruit setting rate, promote fruit growth, and increase single fruit weight and early yield (Yelle et al. 1990). Similarly, Zhao et al. and Zhu et al. showed that increasing the application of $\mathrm{CO}_{2}$ promoted a significant increase in the growth of melon seedlings, along with significant increases in plant height, stem diameter, and leaf area, which are conducive to the cultivation of strong seedlings (Zhu et al. 2004; Zhao et al. 2006). The 
upregulated expression of carbon fixation pathway-associated genes in Guimi melon is assumed to enhance the photosynthetic efficiency of plants (Hao et al. 2011; Li et al. 2014) and promote the accumulation of organic matter and plant growth (Sharwood et al. 2017), whereas an increase in $\mathrm{CO}_{2}$ levels promotes increases in $\mathrm{H}^{+}$concentration in the cell wall, activates enzymes that soften the cell wall, releases the connection of polymers in cell walls, causes relaxation of the cell wall, and contributes to reductions in turgor pressure, thereby resulting in a thickening of leaves and an increase in leaf area (Li et al. 2015). Consequently, photosynthesis-mediated carbon fixation is of importance from the perspective of fruit ripening.

Cell wall metabolism is one of the most important aspects of fruit ripening and is closely associated to flesh texture (Sun et al. 2012), which is a significant quality attribute defining commercial fruit quality (Guo et al. 2013). Plant cell walls play a significant role in determining the shape and elasticity of fruit, and changes in wall structure and composition are considered primary factors contributing to changes in fruit texture (Baldwin et al. 1989). Fruit texture is an important factor affecting fruit quality, storage and transportation characteristics, and disease resistance, and changes in pulp cell wall components are a primary factor contributing to changes in fruit essence (Hobson et al. 1981), whereas fruit softening is positively correlated with the degradation of cell wall components caused by cell wall hydrolases (Rasmussen et al. 1975). Studies have shown that mutations in several key genes involved in plant hormone synthesis and signal transduction can lead to a downregulated expression of cell wall-related genes. For example, the content of aldose and the degree of pectin methyl esterification have been found to be reduced in gibberellin gal-3 and Gai mutants (Derbyshire et al. 2007). The expression of XTH (xyloglucan endotransglucosylase hydrolase) in Arabidopsis hypocotyls has been shown to be induced by gibberellin (Liu et al. 2007), and in the present study, we established that gibberellin metabolic process (GO:0009685), cytoskeleton (G0:0005856), and cell wall organization or biogenesis (G0:0071554) were the most significantly enriched functions in Guimi melon DEG profiles (Fig. 4), thereby indicating that the genes involved in cell wall metabolism might play essential roles in determining fruit ripening. Moreover, we also found that the levels of GUX3 expression underwent a gradual reduction during fruit development. It is currently known that Gux, a glucuronosyltransferase-related gene, plays an important role in the formation of xylan side chains (Bromley et al. 2013; Mortimer et al. 2010), and in Arabidopsis, GUX3 is expressed in roots, stems, leaves and flowers, although only in the xylem. Consequently, it may be involved in the synthesis of certain components of the protowall (Lee et al. 2012), although its associated functions have not been reported to date.

Sugars are essential components of melon fruit quality and serve as prominent signals in the regulation of fruit ripening (Jia et al. 2013; Wu et al. 2014). The soluble sugars found in fruits are primarily sucrose, fructose, and glucose (Lv et al. 2000; Liu et al. 2013), the accumulation of which is closely associated with fruit ripening. Sugars serve as regulatory signals for the expression of numerous genes, among which, sucrose is considered the most effective signal molecule. For example, it has been demonstrated that sucrose functions as a signal molecule that interacts with abscisic acid signaling to regulate strawberry fruit ripening (Jia et al. 2013). In the present study, we identified a number of genes involved in 
sugar metabolism showing differential expression patterns during fruit development, including those associated with 'galactose metabolism', 'starch and sucrose metabolism', 'fructose and mannose metabolism', and 'amino sugar and nucleotide sugar metabolism' (Fig. 6). In the case of several of these genes, we detected marled reductions in expression levels during the middle phase of fruit development, followed by a slight increase in the pre-mature fruit stage, and subsequent decline to the lowest recorded levels of expression in the mature fruit (Fig. 6). The contents of sugar differ according to organ and tissue, and the types of sugar also vary, as in, for example, the vascular bundles of tomato pedicels and fruit stalks, which mainly contain sucrose, whereas in other parts of the fruit, the accumulated sugars are primarily glucose and fructose. Moreover, the contents of glucose and fructose in the pericarp tissue and vascular bundles of tomato fruit differ significantly from those in the pectin and septum (Qi et al. 2001). Similarly, whereas the predominant sugar in grapes is glucose, peaches and apricots accumulate sucrose. These differences are generally determined by the type of sugar metabolism in fruit and the activity of related enzymes (Wang et al. 2009). It has also been established that there are considerable differences with respect sugar type and contents among the different varieties of the same plant, as has been demonstrated in a study of nine white pear varieties (Liu et al. 2015). In the early stages of fruit development, it has been established the sucrose transporter Pu SUT and $\beta$-glucomannases-pubglu1, Pu bglu2, and Pu bglu4-are highly expressed (Li et al. 2016). Furthermore, there have been numerous studies that have examined expression of the sucrose acid invertase gene. For example, Qin et al. (2006) have characterized the cell wall acid invertase gene Mr ivr1 in Myrica rubra, the semi quantitative RT-PCR expression analysis of which revealed highest expression during the early stage of fruit development and relatively low expression in mature fruit. Thus, consistent with the findings of the present study, it has been widely established that the expression of multiple genes associated glucose metabolism differs among different species and varieties of fruit-bearing plants, and also at different stages of fruit ripening.

\section{Conclusion}

In this study, we performed comparative transcriptomic analyses of two varieties of Hami melon (Guimi and Yaolong), with the aim of characterizing the genes and associated pathways controlling the physical and chemical properties of fruits. We successfully identified a number of candidate genes and pathways associated with polysaccharide metabolism, cell wall processes, biosynthesis of secondary metabolites, phenylpropanoid biosynthesis, and carbon fixation, which probably contribute to regulating the size and flavor traits of these fruits. Comparing gene expression patterns at different stages of fruit development in Guimi melons will provide further insights into the regulatory mechanisms underlying the physical and chemical characteristics of melon fruit during the progression of development.

\section{Abbreviations}

DEGs: differentially expressed genes; DAP: days after pollination; KEGG: Kyoto Encyclopedia of Genes and Genomes; GO: Gene Ontology; STEM: short time-series expression miner. 


\section{Declarations}

\section{Ethics approval and consent to participate}

Not applicable.

\section{Consent for publication}

Not applicable.

\section{Availability of data and materials}

The datasets used and/or analysed during the current study are available in the NCBI Bioproject repository, [PRJNA787394].

\section{Competing interests}

The authors declare that they have no competing interests.

\section{Funding}

This work was supported by the Guangxi science and technology base and talent project (GKAD18281095) and Stable funding of scientific research team projects (GNK2021YT169).

\section{Authors' contributions}

R.L. designed the experiments; R.L., Y.S., X.Q., X.L. and J.Z. performed the materials harvest and measurement; Z.G. and Z.F. extracted RNA and prepared the sequencing libraries; R.L., Y.S. and H.Q. analyzed the data; R.L. wrote the manuscript.

\section{References}

1. Abbas HMK, Huang HX, Wang AJ, Wu TQ, Xue SD, Ahmad A, Xie DS, Li JX, Zhong YJ: Metabolic and transcriptomic analysis of two Cucurbita moschata germplasms throughout fruit development. BMC genomics 2020, 21(1).

2. Ashburner M, Ball CA, Blake JA, Botstein D, Butler H, Cherry JM, Davis AP, Dolinski K, Dwight SS, Eppig JT et al: Gene Ontology: tool for the unification of biology. Nature genetics 2000, 25(1):25-29.

3. Baldwin EA, Pressey R: Pectic enzymes in pectolyase: separation, characterization, and induction of ethylene in fruits. Plant physiology 1989, 90(1):191-196.

4. Bi Y, Tian SP, Lui HX, Zhao J, Cao JK, Li YC, Zhang WY: Effect of temperature on chilling injury, decay and quality of Hami melon during storage. Postharvest Biol Tec 2003, 29(2):229-232.

5. Braun DM: SWEET! The Pathway Is Complete. Science 2012, 335(6065):173-174. 
6. Bromley JR, Busse-Wicher M, Tryfona T, Mortimer JC, Zhang ZN, Brown DM, Dupree P: GUX1 and GUX2 glucuronyltransferases decorate distinct domains of glucuronoxylan with different substitution patterns. Plant J 2013, 74(3):423-434.

7. Burger Y, Sa'ar U, Distelfeld A, Katzir N, Yeselson Y, Shen S, Schaffer AA: Development of sweet melon (Cucumis melo) genotypes combining high sucrose and organic acid content. J Am Soc Hortic Sci 2003, 128(4):537-540.

8. Burger $Y$, Schaffer AA: The contribution of sucrose metabolism enzymes to sucrose accumulation in Cucumis melo. J Am Soc Hortic Sci 2007, 132(5):704-712.

9. Chen SF, Zhou YQ, Chen YR, Gu J: fastp: an ultra-fast all-in-one FASTQ preprocessor. Bioinformatics 2018, 34(17):884-890.

10. Colla G, Roupahel Y, Cardarelli M, Rea E: Effect of salinity on yield, fruit quality, leaf gas exchange, and mineral composition of grafted watermelon plants. Hortscience 2006, 41(3):622-627.

11. Dardick CD, Callahan AM, Chiozzotto R, Schaffer RJ, Piagnani MC, Scorza R: Stone formation in peach fruit exhibits spatial coordination of the lignin and flavonoid pathways and similarity to Arabidopsis dehiscence. Bmc Biology 2010, 8.

12. Derbyshire P, McCann MC, Roberts K: Restricted cell elongation in Arabidopsis hypocotyls is associated with a reduced average pectin esterification level. Bmc Plant Biol 2007, 7.

13. Ernst J, Bar-Joseph Z: STEM: a tool for the analysis of short time series gene expression data. BMC bioinformatics 2006, 7 .

14. Grassi S, Piro G, Lee JM, Zheng Y, Fei ZJ, Dalessandro G, Giovannoni JJ, Lenucci MS: Comparative genomics reveals candidate carotenoid pathway regulators of ripening watermelon fruit. BMC genomics 2013, 14.

15. Guo SG, Sun HH, Zhang HY, Liu JA, Ren Y, Gong GY, Jiao C, Zheng Y, Yang WC, Fei ZJ et al: Comparative Transcriptome Analysis of Cultivated and Wild Watermelon during Fruit Development. PloS one $2015,10(6)$.

16. Hubbard NL, Huber SC, Pharr DM: Sucrose Phosphate Synthase and Acid Invertase as Determinants of Sucrose Concentration in Developing Muskmelon (Cucumis-Melo L) Fruits. Plant physiology 1989, 91(4):1527-1534.

17. Jia HF, Wang YH, Sun MZ, Li BB, Han Y, Zhao YX, Li XL, Ding N, Li C, Ji WL et al: Sucrose functions as a signal involved in the regulation of strawberry fruit development and ripening. New Phytol 2013, 198(2):453-465.

18. Kim D, Landmead B, Salzberg SL: HISAT: a fast spliced aligner with low memory requirements. Nature methods 2015, 12(4):357-U121.

19. Langmead B, Salzberg SL: Fast gapped-read alignment with Bowtie 2. Nature methods 2012, 9(4):357-U354.

20. Lee C, Zhong RQ, Ye ZH: Arabidopsis Family GT43 Members are Xylan Xylosyltransferases Required for the Elongation of the Xylan Backbone. Plant Cell Physiol 2012, 53(1):135-143. 
21. Li HD, Wang Y, Xiao J, Xu K: Reduced photosynthetic dark reaction triggered by ABA application increases intercellular $\mathrm{CO} 2$ concentration, generates $\mathrm{H} 2 \mathrm{O} 2$ and promotes closure of stomata in ginger leaves. Environmental and experimental botany 2015, 113:11-17.

22. Li Z, Zhang Y, Yu D, Zhang N, Lin J, Zhang J, Tang J, Wang J, Mu C: The influence of precipitation regimes and elevated $\mathrm{CO} 2$ on photosynthesis and biomass accumulation and partitioning in seedlings of the rhizomatous perennial grass Leymus chinensis. PloS one 2014, 9(8):e103633.

23. Liu JA, Guo SG, He HJ, Zhang HY, Gong GY, Ren Y, Xu Y: Dynamic characteristics of sugar accumulation and related enzyme activities in sweet and non-sweet watermelon fruits. Acta Physiol Plant 2013, 35(11):3213-3222.

24. Liu YB, Lu SM, Zhang JF, Liu S, Lu YT: A xyloglucan endotransglucosylase/hydrolase involves in growth of primary root and alters the deposition of cellulose in Arabidopsis. Planta 2007, 226(6):1547-1560.

25. Ma J, Wang J, Ghoraie LS, Men X, Chen R, Dai PG: Comprehensive expression-based isoform biomarkers predictive of drug responses based on isoform co-expression networks and clinical data. Genomics 2020, 112(1):647-658.

26. Mortimer JC, Miles GP, Brown DM, Zhang ZN, Segura MP, Weimar T, Yu XL, Seffen KA, Stephens E, Turner SR et al: Absence of branches from xylan in Arabidopsis gux mutants reveals potential for simplification of lignocellulosic biomass. Proceedings of the National Academy of Sciences of the United States of America 2010, 107(40):17409-17414.

27. Nunez-Palenius HG, Gomez-Lim M, Ochoa-Alejo N, Grumet R, Lester G, Cantliffe DJ: Melon fruits: Genetic diversity, physiology, and biotechnology features. Crit Rev Biotechnol 2008, 28(1):13-55.

28. Pruitt KD, Tatusova T, Maglott DR: NCBI Reference Sequence (RefSeq): a curated non-redundant sequence database of genomes, transcripts and proteins. Nucleic acids research 2005, 33:D501D504.

29. Rasmussen GK: Cellulase Activity, Endogenous Abscisic Acid, and Ethylene in Four Citrus Cultivars during Maturation. Plant physiology 1975, 56(6):765-767.

30. Saladie M, Canizares J, Phillips MA, Rodriguez-Concepcion M, Larrigaudiere C, Gibon Y, Stitt M, Lunn $\mathrm{JE}$, Garcia-Mas J: Comparative transcriptional profiling analysis of developing melon (Cucumis melo L.) fruit from climacteric and non-climacteric varieties. BMC genomics 2015, 16:440.

31. Schaffer AA, Aloni B, Fogelman E: Sucrose Metabolism and Accumulation in Developing Fruit of Cucumis. Phytochemistry 1987, 26(7):1883-1887.

32. Sharwood RE, Crous KY, Whitney SM, Ellsworth DS, Ghannoum O: Linking photosynthesis and leaf N allocation under future elevated $\mathrm{CO} 2$ and climate warming in Eucalyptus globulus. J Exp Bot 2017, 68(5):1157-1167.

33. Sun L, Sun Y, Zhang M, Wang L, Ren J, Cui M, Wang Y, Ji K, Li P, Li Q et al: Suppression of 9-cisepoxycarotenoid dioxygenase, which encodes a key enzyme in abscisic acid biosynthesis, alters fruit texture in transgenic tomato. Plant physiology 2012, 158(1):283-298. 
34. Thirunavukkarasu N, Sharma R, Singh N, Shiriga K, Mohan S, Mittal S, Mittal S, Mallikarjuna MG, Rao AR, Dash PK et al: Genomewide Expression and Functional Interactions of Genes under Drought Stress in Maize. Int J Genomics 2017, 2017.

35. Trapnell C, Williams BA, Pertea G, Mortazavi A, Kwan G, van Baren MJ, Salzberg SL, Wold BJ, Pachter L: Transcript assembly and quantification by RNA-Seq reveals unannotated transcripts and isoform switching during cell differentiation. Nature biotechnology 2010, 28(5):511-U174.

36. Villanueva MJ, Tenorio MD, Esteban MA, Mendoza MC: Compositional changes during ripening of two cultivars of muskmelon fruits. Food Chem 2004, 87(2):179-185.

37. Wang J, Mao LC, Li XW, Lv Z, Liu CH, Huang YY, Li DD: Oxalic acid pretreatment reduces chilling injury in Hami melons (Cucumis melo var. reticulatus Naud.) by regulating enzymes involved in antioxidative pathways. Sci Hortic-Amsterdam 2018, 241:201-208.

38. Wu JX, Xu ZL, Zhang YJ, Chai LJ, Yi HL, Deng XX: An integrative analysis of the transcriptome and proteome of the pulp of a spontaneous late-ripening sweet orange mutant and its wild type improves our understanding of fruit ripening in citrus. J Exp Bot 2014, 65(6):1651-1671.

39. Xu GL, Singh SK, Reddy VR, Barnaby JY, Sicher RC, Li T: Soybean grown under elevated CO2 benefits more under low temperature than high temperature stress: Varying response of photosynthetic limitations, leaf metabolites, growth, and seed yield. J Plant Physiol 2016, 205:20-32.

40. Yamaguchi M, Hughes DL, Yabumoto K, Jennings WG: Quality of Cantaloupe Muskmelons Variability and Attributes. Sci Hortic-Amsterdam 1977, 6(1):59-70.

41. Yu KQ, Xu Q, Da XL, Guo F, Ding YD, Deng XX: Transcriptome changes during fruit development and ripening of sweet orange (Citrus sinensis). BMC genomics 2012, 13.

42. Zenda T, Liu ST, Wang X, Liu G, Jin HY, Dong AY, Yang YT, Duan HJ: Key Maize Drought-Responsive Genes and Pathways Revealed by Comparative Transcriptome and Physiological Analyses of Contrasting Inbred Lines. International journal of molecular sciences 2019, 20(6).

43. Zhang H, Wang HS, Yi HP, Zhai WQ, Wang GZ, Fu QS: Transcriptome profiling of Cucumis melo fruit development and ripening. Hortic Res-England 2016, 3.

44. Zhu QL, Gao P, Liu S, Zhu ZC, Amanullah S, Davis AR, Luan FS: Comparative transcriptome analysis of two contrasting watermelon genotypes during fruit development and ripening. BMC genomics 2017, 18.

45. Alan O, Ozdemir N, Gunen Y. Effect of grafting on watermelon plant growth, yield and quality. Journal of Agronomy, 2007, 6: 362.

46. Schaffer AA, Pharr DM, Madore MA. Cucurbits. In: Zamski E, Schaffer AA (eds.). Photoassimilate distribution in plants and crops. New York: CRC Press, 1996;729-757.

47. Yelle S, Beeson RC, Jr, Trudel MJ. Duration of $\mathrm{CO} 2$ enrichment influences growth, yield, and gasexchange of two tomato species. Journal of the American Society for Horticultural Science, 1990,115: 52-57.

48. Zhao FY. Physiological effects of $\mathrm{CO} 2$ enrichment on melon seedlings in Greenhous. Agricultural University of Anhui, 2006. 
49. Zhu SD, Xu WJ, Zhao FY. Physiological effects of CO2 enrichment on watermelon and melon seedlings in Greenhouse. Journal of Huazhong Agricultural University, 2004(z2): 287-291.

50. Hao XY, Han X, Li P, et al. Effects of elevated atmospheric $\mathrm{CO} 2$ concentration on mung bean leafphotosynthesis and chlorophyll fluorescence parameters. The journal of applied ecology, 2011, 22(10): 2776-2780.

51. Hobson GE. Enzymes and texture changes during ripening. In: Recent Advances in the Biochemistry of Fruits and Vegetables. London: Academic Press, 1981:123-132.

52. Lv XM, Zhang DP. Accumulation of sugars in developing fruits. Plant Physiology Communication, 2000, 36(3): 258-265.

53. Liu X, Gong RG, Zhang X. The activity analysis for sugar-metabolizining related enzymes in 3 kinds of navel orange fruits. Journal of Northwest A\&F University, 2013, 41(5): 136-140.

54. Qi HY, Li TL, Zou LN. Changes of composition and content of carbohydrate during tomato fruit development. Journal of Shenyang Agricultural University, 2001, 32(5): 346-348.

55. Wang C, Fang JG, Wang T. The sugar metabolism in fruits. Acta Agriculturae Zhejiangensis, 2009, 21(5): 529-534.

56. Liu SZ, Liu J, Zhang Y. Mass fraction of sugar and acid and flavor index of pear cultivars (Pyurs) ripening stages. Acta Agriculturae Boreali-occidentalis Sinica, 2015, 24(1): 97-102.

57. Li XY, Li T, Yuan H. Sugar accumulation and expression of related genes in'Nanguo'pear fruit. Journal of Fruit Science, 2016, 33(s1): 59-64.

58. Qin QP, Chen JW, Cheng JH. Isolation and expression analysis of acid invertase gene cDNA from Myrica rubra. Journal of Fruit Science, 2006, 23(4): 558-561.

\section{Figures}


5 DAP

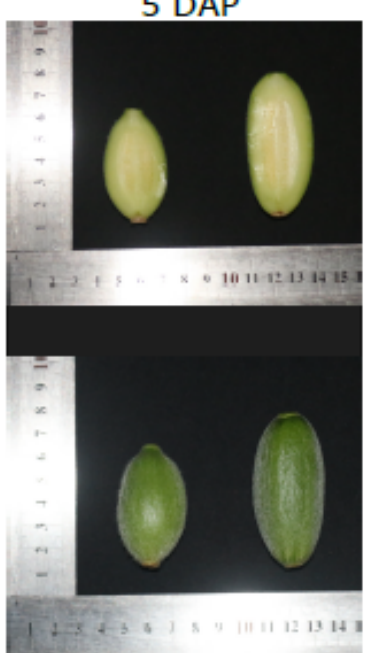

25 DAP

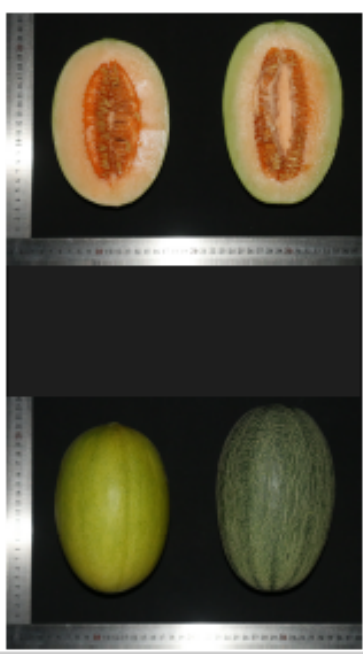

10 DAP

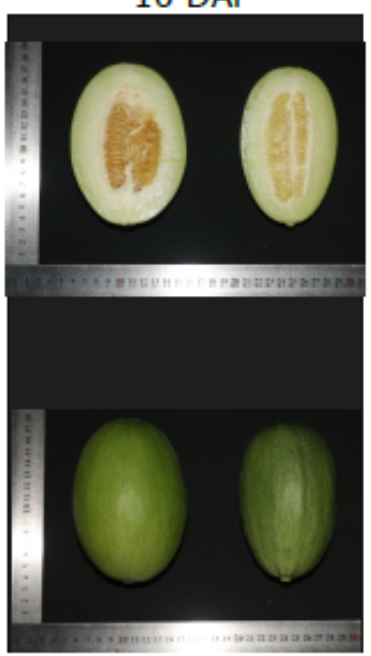

30 DAP

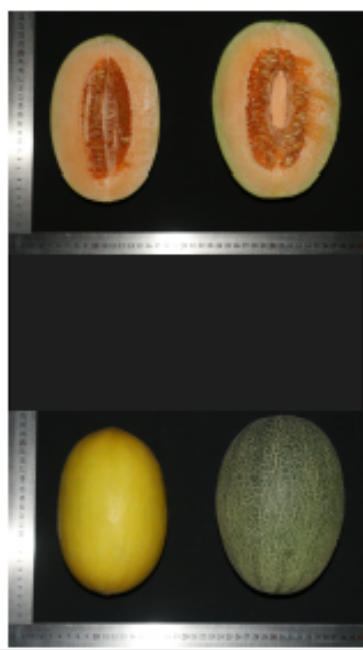

15 DAP

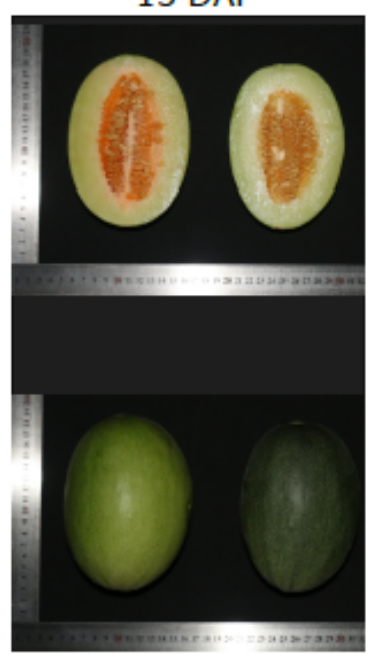

35 DAP

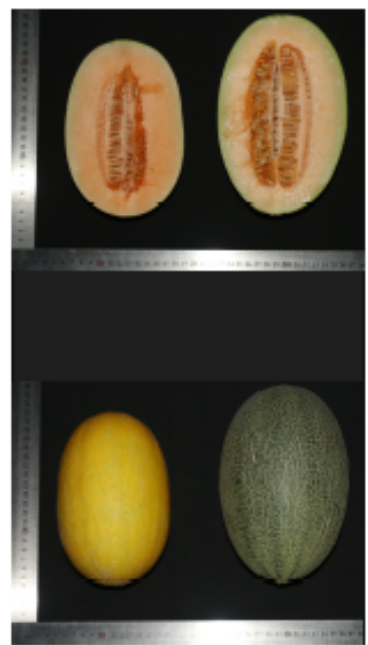

20 DAP

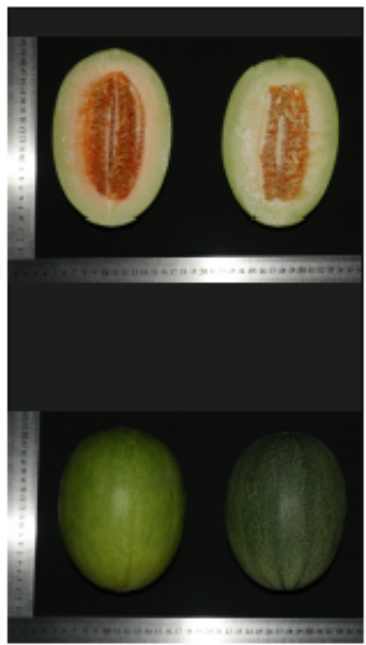

40 DAP

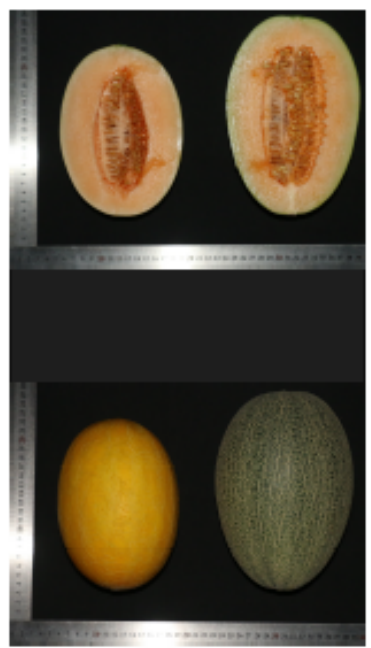

\section{Figure 1}

Fruits of the Guimi and Yaolong varieties of Hami melon at different stages of development. For each sampling time image, Guimi and Yaolong fruits are depicted on the left- and right-hand sides, respectively.
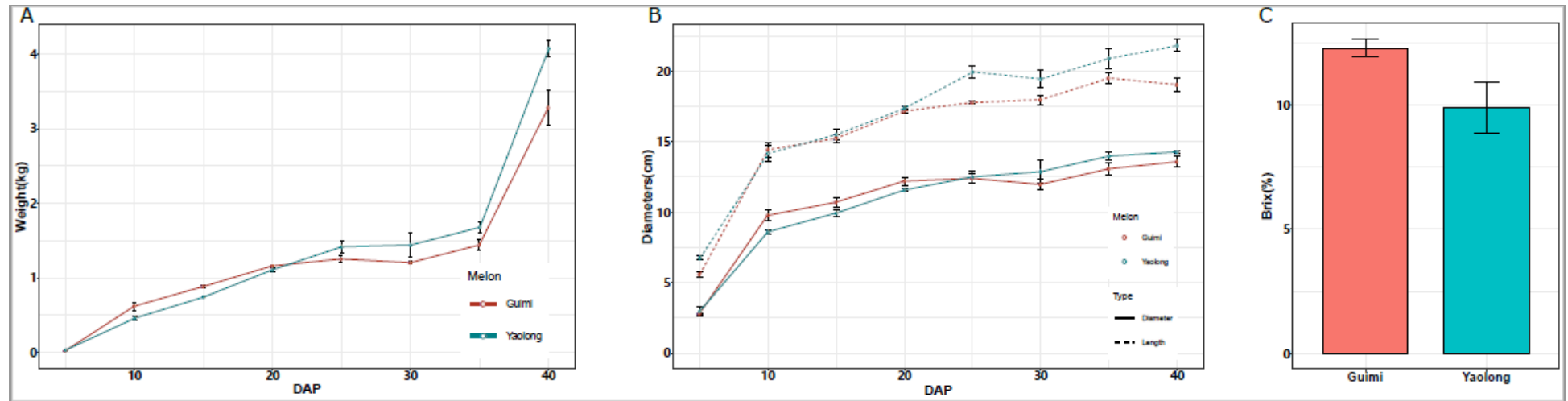

Figure 2

Differences in the weight, size, and soluble sugar contents of fruits of the Guimi and Yaolong varieties of Hami melon. Trends in weight (A) and size (length, diameter) (B) at 5, 10, 15, 20, 25, 30, 35, and 40 days 
after pollination (DAP). (C) Total soluble sugar contents at 40 DAP. Data represent the means of three individual replicates. The bars denote standard error values $(n=3)$.

A

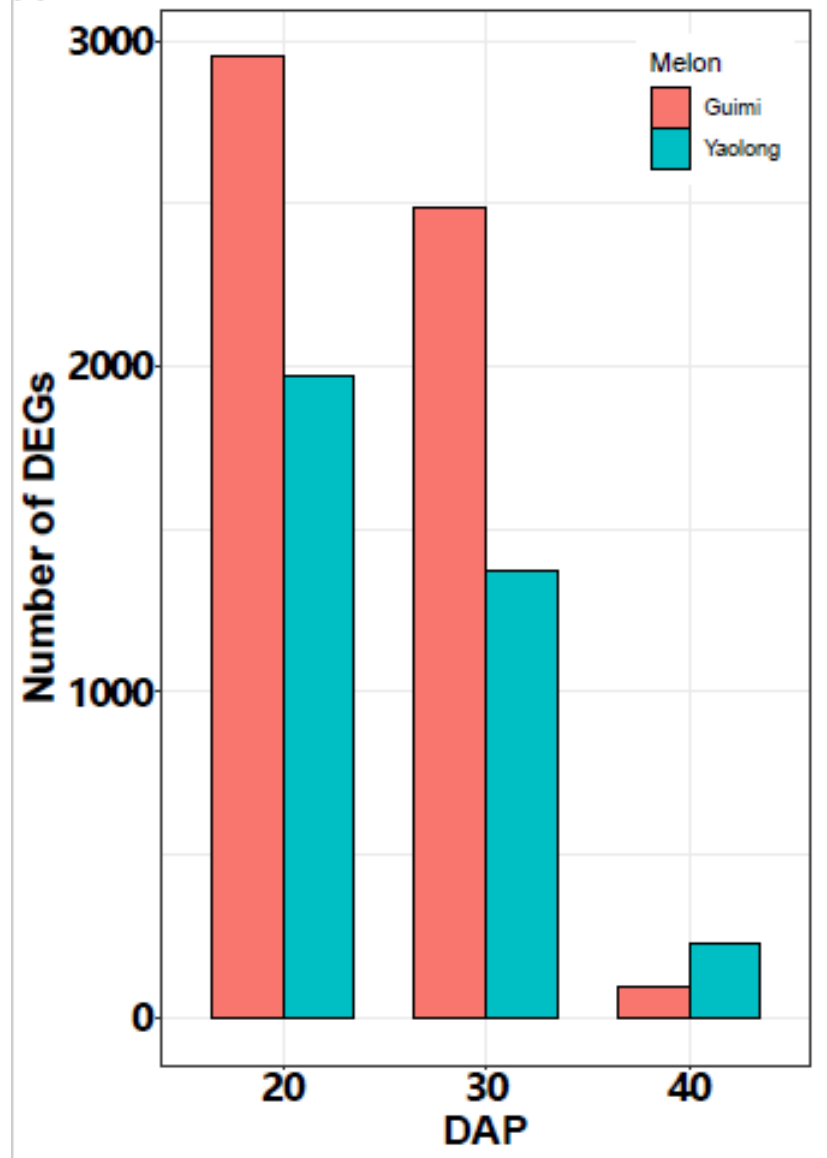

B

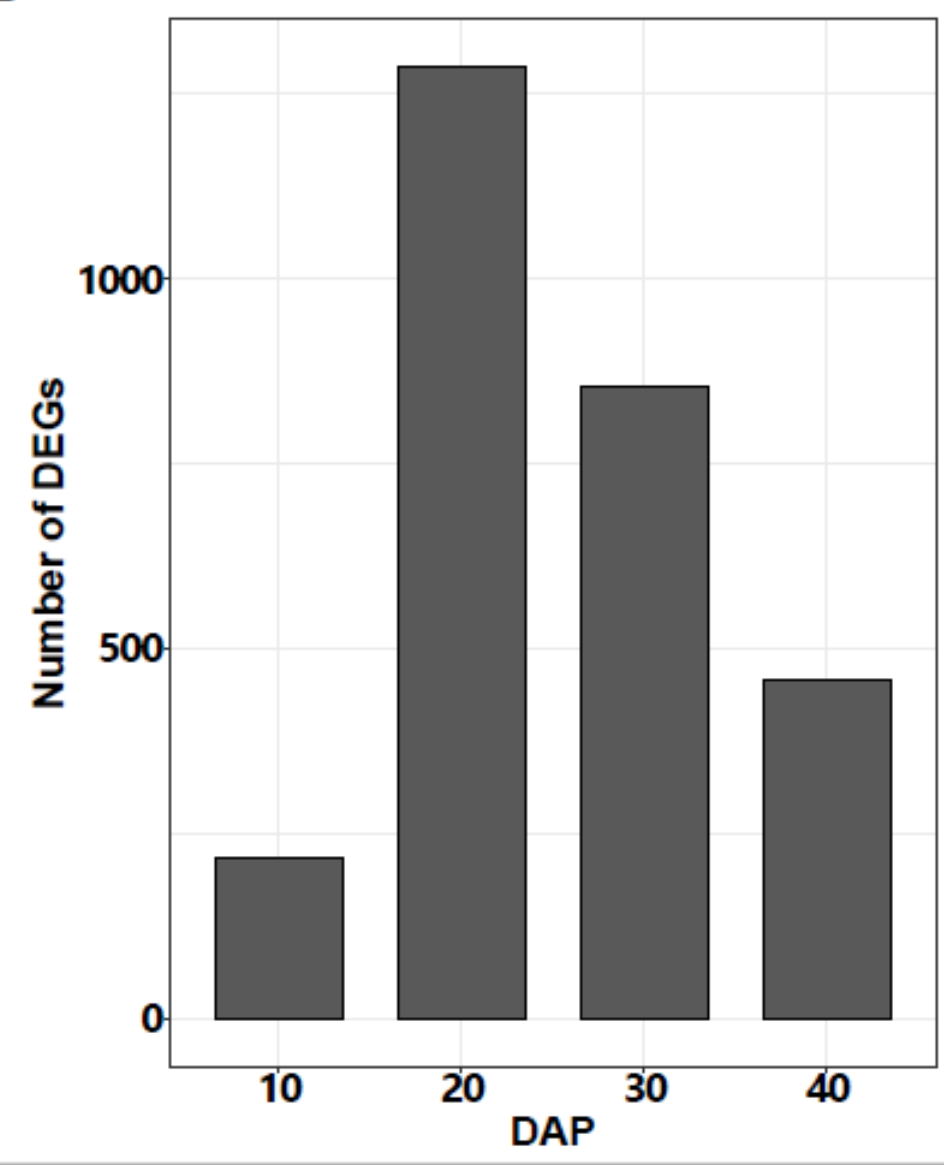

Figure 3

Distribution of differentially expressed genes (DEGs) at different time points after pollination during melon fruit development and ripening. (A) The number of DEGs in different samples. Light coral and medium turquoise colors denote DEGs in the Guimi and Yaolong varieties, respectively. (B) Distribution of DEGs between Guimi and Yaolong at specific sampling times. 
A

Up
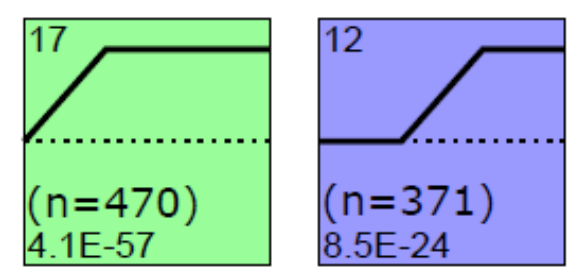

$\mathrm{Bi}$

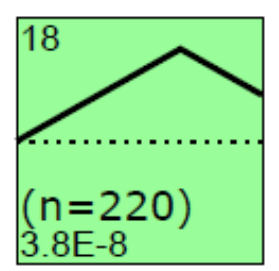

Down

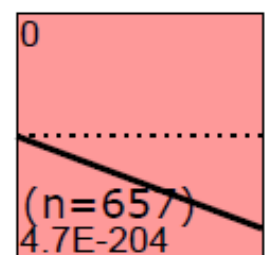

B
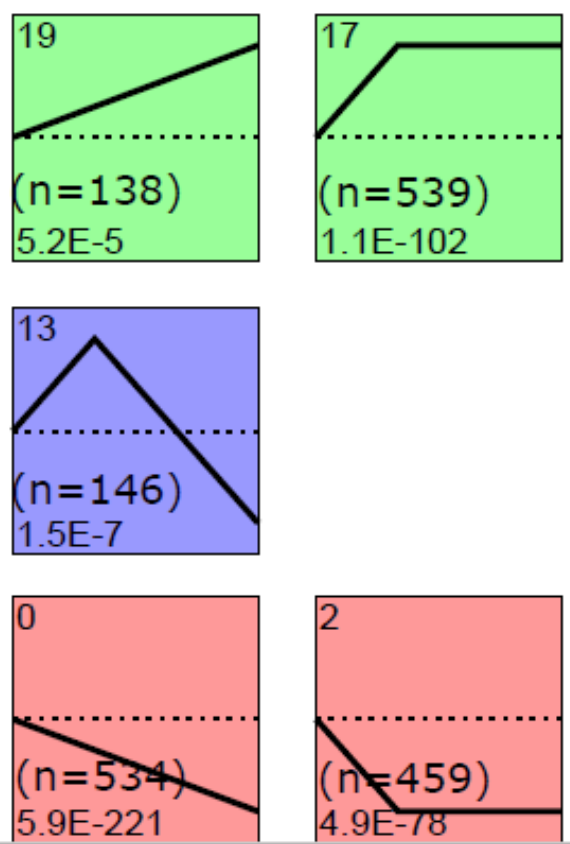

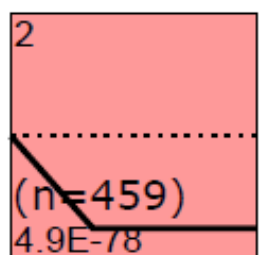

$4.9 \mathrm{E}-18$

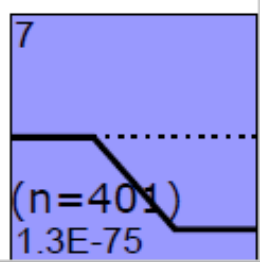

\section{Figure 4}

Enriched profiles of differentially expressed genes (DEGs) during fruit development. Profiles of Guimi. (A) and Yaolong (B) were clustered into three groups, namely Up (upregulated), Bi (biphasic expression pattern), and Down (downregulated). Profile numbers are indicated at the top left-hand corner, and the corresponding P-values for each profile are shown at the bottom left-hand corner. The number DEGs within each profile is shown in brackets. 

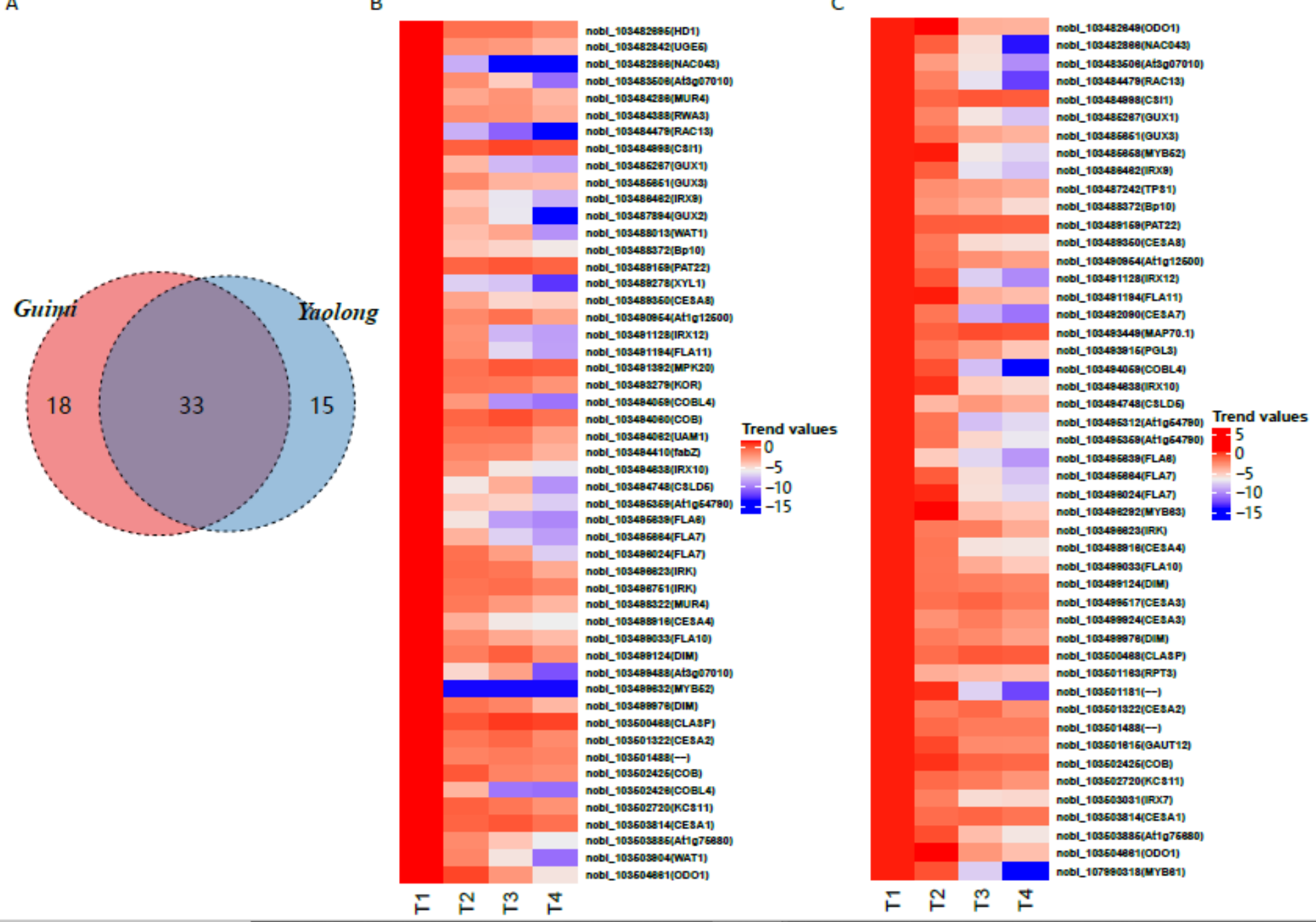

\section{Figure 5}

Trends in the expression changes of key genes associated with cell wall biogenesis in two Hami melon varieties. (A) Common differentially expressed genes (DEGs) in the cell wall biogenesis pathway of the two varieties. DEGs for Guimi (B) and Yaolong (C). 
A

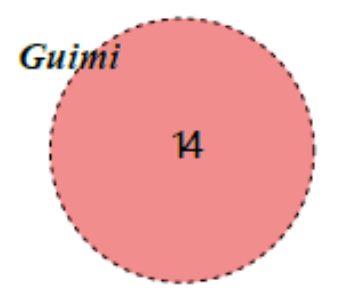

B

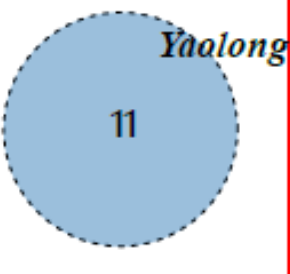

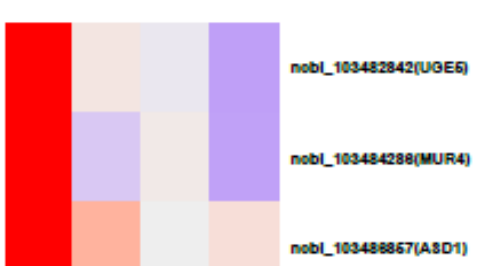

nobl_10948s477(UOOD)

nobl_103490928(GAE2)

nobl_103492828(GAUTE)

nobl_103492904(UOBD1)

nObl_103483600(GER2)

nobl_109495448(Xy11)

nobl_10548s117(GLCAK1)

nobl_109601607(GAUT7)

nobl_108601836(UX86)

nobl_10860373a(ARA1)

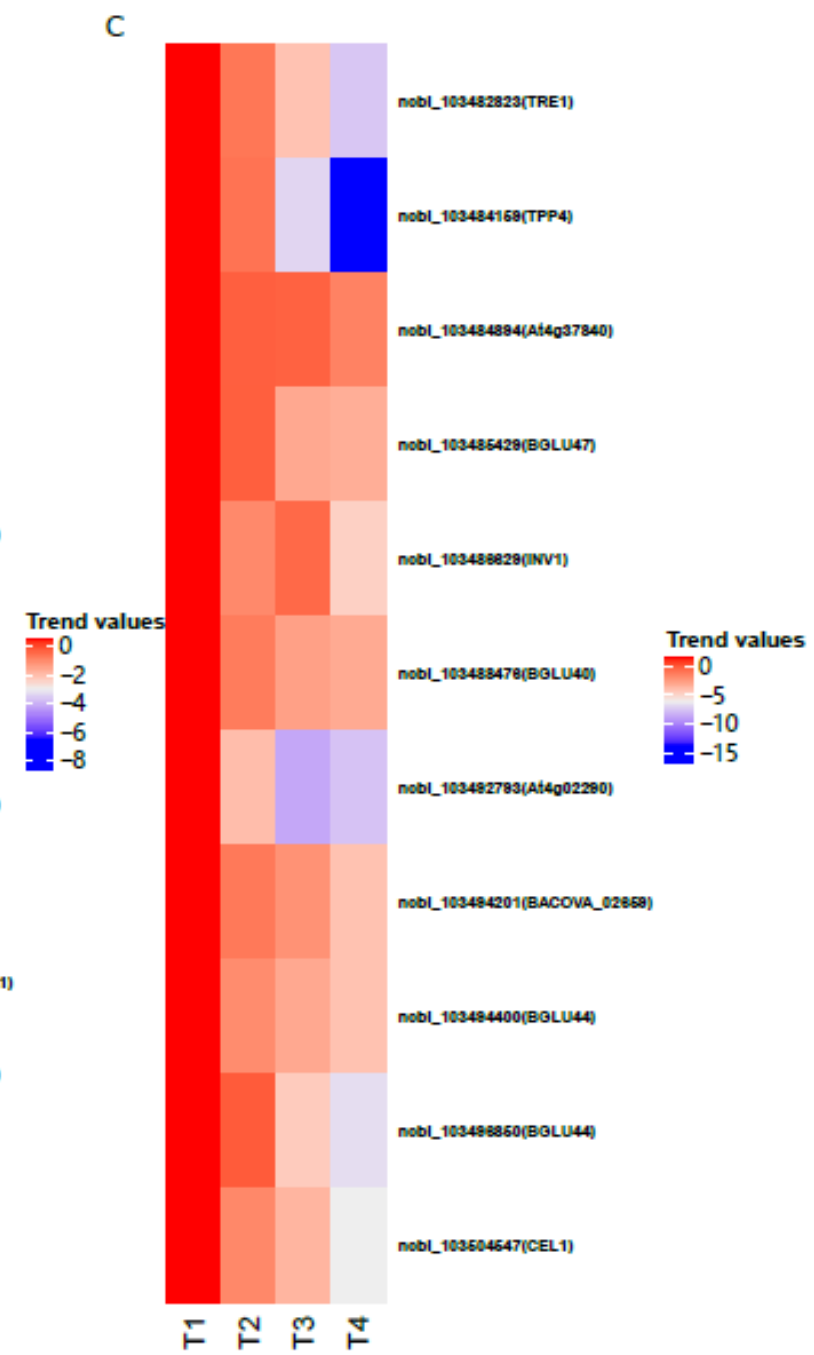

\section{Figure 6}

Trends in the expression changes of key genes associated with sugar metabolism pathways in the two Hami melon varieties. (A) The number of differentially expressed genes (DEGs) in sugar metabolism pathways in the two varieties. Expression trends of DEGs in Guimi (B) and Yaolong (C).

\section{Supplementary Files}

This is a list of supplementary files associated with this preprint. Click to download.

- FigureS1.pdf

- Figures2.pdf

- FigureS3.pdf

- FigureS4.pdf

- Figures5.pdf

- FigureS6.pdf 
- FigureS7.pdf

- FigureS8.pdf

- Supplementarytables.docx 